\title{
Active Self-Sensing Scheme Development for Structural Health Monitoring
}

\author{
Sang Jun Lee \& Hoon Sohn \\ The Department of Civil and Environmental Engineering, Carnegie Mellon University, \\ Pittsburgh, PA 15213, USA \\ sangjunl@andrew.cmu.edu; $\underline{\text { hsohn@cmu.edu }}$
}

\begin{abstract}
Smart materials such as Lead Zirconate Titanate (PZT) have been widely used for generating and measuring guided waves in solid media for structural health monitoring (SHM) applications. In this study, a self-sensing system, composed of self-sensing algorithms and a self-sensing circuit, is developed so that a single PZT wafer can be used for simultaneous actuation and sensing. First, a PZT wafer is modeled as a single capacitor and a voltage source, and the proposed self-sensing scheme is calibrated by applying a probing waveform. Then, the mechanical response of the PZT wafer coupled with the target structure is extracted from the measured PZT output voltage when an arbitrary excitation is applied to the same PZT wafer. While existing self-sensing techniques focus on vibration controls, the proposed self-sensing scheme attempts to improve the accuracy of extracted sensing signals in the time domain. The simplicity, adaptability and autonomous nature of the proposed selfsensing scheme make it attractive for continuous monitoring of structures in field. The effectiveness of the proposed self-sensing scheme is investigated through experiments of a cantilever beam.
\end{abstract}

\section{Introduction}

Structural Health Monitoring (SHM) and Non-Destructive Testing (NDT) is an integrated process of sensing, information technology and statistical inference used to ensure the safety and performance of a structure and to provide early detection of critical damage. Recently, guided waves using wafer-type piezoelectric materials such as Lead Zirconate Titanate (PZT) have been widely used for damage detection because they have a sensing range, which is in-between of those conventional NDT techniques and global SHM techniques (Sohn et al., 2004). One of the important characteristics of the PZT materials is that it can be used for simultaneous sensing and actuation. This feature of the PZT materials is called self-sensing here, and the advantages of a self-sensing scheme include: (1) the pulse-echo time reversal method can be achieved using only a single PZT wafer (Fink, 1999), (2) sensor diagnosis schemes can be implemented based on self-sensing to monitor sensors' performance and integrity (Lee et al.,2006), (3) a single PZT wafer can be used to suppress undesired vibrations (Cole and Clark, 1994; Dosch et al., 1992; Vipperman and Clark, 1996). The ultimate goal of this study is to apply self-sensing to guided wave propagation. Therefore, this study focuses on estimation of the mechanical response in the time domain. The proposed self-sensing fully takes advantage of the facts that any user-defined input signals can be applied to a structure and the input waveform is known. Additional advantages of the proposed approach are its simplicity and adaptability. Only additional hardware required is a self-sensing circuit equivalent to a charge amplifier, and the self-sensing parameters can be calibrated instantaneously at the presence of changing operational and environmental conditions of the system. This paper is organized as follows: First, the proposed self-sensing scheme is theoretically developed. Then, experimental studies are performed to demonstrate the 
effectiveness of the proposed self-sensing scheme. Finally, this paper concludes with a brief summary and discussions for future work.

\section{Theoretical Development}

This section develops the theoretical framework of the proposed self-sensing scheme. Figure 1 shows the schematic of the proposed self-sensing circuit and a cantilever beam used in this study and an actual experimental set-up. A single PZT is mounted on one surface of the beam near the fixed end. The proposed self-sensing scheme consists of two main steps: (1) calibration of the self-sensing circuit by applying a probing waveform and (2) extraction of a mechanical response corresponding to an arbitrary input waveform. The first step (step I) is to measure a so-called scaling factor, which is defined as the ratio of the PZT capacitance to that of the feedback capacitor in the self-sensing circuit. The proposed scaling factor is estimated by three different algorithms: root mean squares (RMS), least mean squares (LMS) and orthogonality algorithms. In the second step (step II), the mechanical response corresponding to an arbitrary input signal is estimated using the previously calibrated self-sensing circuit.

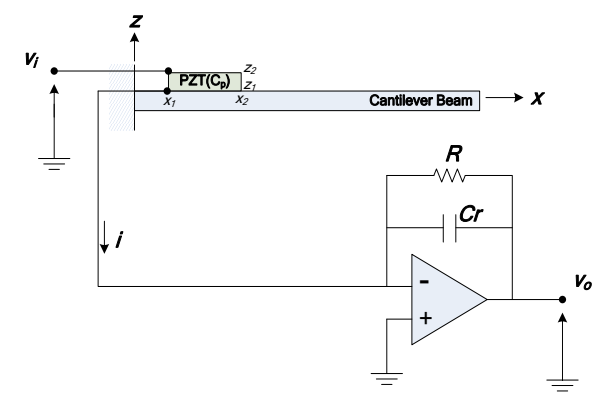

(a) Schematic of the self-sensing circuit

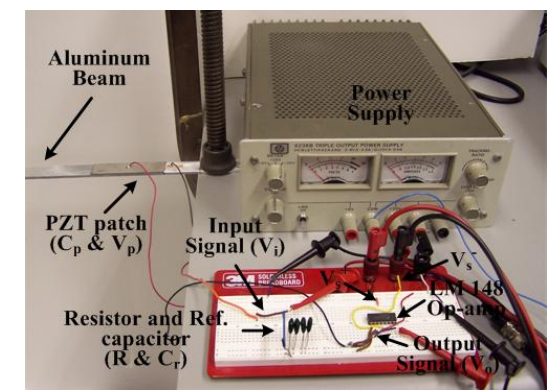

(b) A prototype of the self-sensing scheme

Figure 1. A sensor self-sensing and sensor-diagnosis circuit is being developed to use a single PZT for simultaneously actuation and sensing

\section{Circuit model with PZT wafer}

The output voltage of the self-sensing circuit is related to the input and mechanical response voltages of the PZT. From the circuit diagram shown in figure 1 (a), the output of the circuit is related to the input and mechanical voltages of the PZT as:

$$
\begin{gathered}
i(t)=C_{p}\left[\dot{v}_{i}(t)+\dot{v}_{p}(t)\right]=-C_{r} \dot{v}_{o}(t)-\frac{v_{o}(t)}{R} \\
\dot{v}_{o}(t)+\frac{v_{o}(t)}{R C_{r}}=-\frac{C_{p}}{C_{r}}\left[\dot{v}_{i}(t)+\dot{v}_{p}(t)\right]
\end{gathered}
$$

where $C_{p}, R$ and $C_{r}$ are the PZT capacitance, the feedback resistance and the feedback capacitance of the self-sensing circuit, respectively. When a sinusoidal input $v_{i}(t)=V \sin (\omega t)$ is applied to the PZT wafer, the steady-state solution of (2) becomes under the assumption that the mechanical response is negligible:

$$
v_{o}(t)=-\frac{C_{p}}{C_{r}} \frac{\omega^{2} R^{2} C_{r}^{2}}{\omega^{2} R^{2} C_{r}^{2}+1} V \sin \omega t-\frac{C_{p}}{C_{r}} \frac{\omega R C_{r}}{\omega^{2} R^{2} C_{r}^{2}+1} V \cos \omega t
$$

As the driving frequency $\omega$ increases, the relationship between the output and input voltages can be simplified: 


$$
v_{o}(t) \square-\frac{C_{p}}{C_{r}} V \sin \omega t=-\frac{C_{p}}{C_{r}} v_{i}(t)
$$

where the scaling factor of the proposed self-sensing circuit is defined as:

$$
S F=-\frac{C_{p}}{C_{r}} \square \frac{v_{o}(t)}{v_{i}(t)}
$$

The scaling factor can be approximated by computing the amplitude ratio of the output voltage to that of the input voltage when the driving frequency is high enough.

\section{Scaling factor estimation algorithms}

In this subsection, three different algorithms for estimating the scaling factor are derived and their susceptibility to Gaussian white noise in the input and output voltages is investigated. First, the scaling factor is estimated by computing the ratio of the RMS value of the output signal to that of the input signal as follows:

$$
\begin{aligned}
S F_{R M S} & =-\frac{R M S\left\{\tilde{v}_{o}[k]\right\}}{R M S\left\{\tilde{v}_{i}[k]\right\}}=-\frac{\sqrt{\frac{1}{m} \sum_{k=0}^{m-1}\left(v_{o}[k]+e_{o}[k]\right)^{2}}}{\sqrt{\frac{1}{m} \sum_{k=0}^{m-1}\left(v_{i}[k]+e_{i}[k]\right)^{2}}} \\
& =-\sqrt{\sum_{k=0}^{m-1}\left\{\left(-\frac{C_{p}}{C_{r}} \cdot\left(v_{i}[k]+v_{p}[k]\right)\right)^{2}+e_{o}^{2}[k]\right\} / \sum_{k=0}^{m-1}\left(v_{i}^{2}[k]+e_{i}^{2}[k]\right)}
\end{aligned}
$$

where $\tilde{v}_{o}[k]$ and $\tilde{v}_{i}[k]$ denote noise-contaminated versions of the discrete input and output signals and defined as $\tilde{v}_{o}[k]=v_{o}[k]+e_{o}[k]$ and $\tilde{v}_{i}[k]=v_{i}[k]+e_{i}[k]$, respectively. $e_{o}[k]$ and $e_{i}[k]$ are output and input Gaussian white noises. $m$ is the total length of the measured discrete time signal. Note that, because $e_{i}[k]$ is a Gaussian white noise and independent of the input signal, the sum of $v_{i}[k] \cdot e_{i}[k]$ in (6) converges to zero as the sample number $m$ increases. However, the RMS terms of the input and output noises remain in (6).

Second, from the previous work by Cole and Clark (1994), a LMS algorithm is applied to estimate the scaling factor by calculating the ratio of the cross correlation between the output and input signals to the autocorrelation of the input signal as:

$$
\begin{aligned}
S F_{L M S}= & \frac{E\left\{\tilde{v}_{o}[k] \cdot \tilde{v}_{i}[k]\right\}}{E\left\{\tilde{v}_{i}[k] \cdot \tilde{v}_{i}[k]\right\}}=\frac{E\left\{\left(v_{o}[k]+e_{o}[k]\right) \cdot\left(v_{i}[k]+e_{i}[k]\right)\right\}}{E\left\{\left(v_{i}[k]+e_{i}[k]\right) \cdot\left(v_{i}[k]+e_{i}[k]\right)\right\}} \\
= & \frac{-\frac{C_{p}}{C_{r}} \cdot\left(E\left\{v_{i}^{2}[k]\right\}+E\left\{v_{i}[k] \cdot v_{p}[k]\right\}\right)}{E\left\{v_{i}^{2}[k]\right\}+E\left\{e_{i}^{2}[k]\right\}}
\end{aligned}
$$

where $E\{\}$ is the expectation operator. Because the input and output noises are assumed to be Gaussian white noises, they are independent of the output and input voltages as well as each other. Furthermore, by choosing a high-frequency input signal, the mechanical response can be minimized and the error due to the cross correlation term $E\left\{v_{i}[k] v_{p}[k]\right\}$ becomes negligible. Therefore, only the error due to the Gaussian input white noise remains in the denominator of (7).

Third, in the orthogonality method, the numerator and denominator in (5) are first multiplied by a sinusoidal wave at the input frequency. Then, the numerator and 
denominator are summed over the entire length of the signal as follows:

$$
\begin{aligned}
S F_{\text {ORT }}= & \frac{\sum_{k=0}^{m-1} \tilde{v}_{o}[k] \cdot \sin (\omega k \Delta t)}{\sum_{k=0}^{m-1} \tilde{v}_{i}[k] \cdot \sin (\omega k \Delta t)}=\frac{\sum_{k=0}^{m-1}\left\{-\frac{C_{p}}{C_{r}}\left(v_{i}[k]+v_{p}[k]\right)+e_{o}[k]\right\} \cdot \sin (\omega k \Delta t)}{\sum_{k=0}^{m-1}\left(v_{i}[k]+e_{o}[k]\right) \cdot \sin (\omega k \Delta t)} \\
= & \frac{\sum_{k=0}^{m-1}\left\{-\frac{C_{p}}{C_{r}}\left(v_{i}[k]+v_{p}[k]\right)\right\} \cdot \sin (\omega k T)}{\sum_{k=0}^{m-1} v_{i}[k] \cdot \sin (\omega k T)}
\end{aligned}
$$

Since the orthogonality algorithm uses the ideal sinusoidal signal that doesn't have a noise term, the orthogonality method is expected to be less susceptible to input and output noises. The cross correlation term $E\left\{v_{p}[k] \cdot \sin (\omega k \Delta t)\right\}$ in (8) remains insignificant as long as the amplitude of $v_{p}[k]$ at the driving frequency is negligible. Note that the response component of $v_{p}[k]$ outside the driving frequency is cancelled out through the orthogonality of trigonometric signals. The theoretical analysis suggests that the orthogonality algorithm has the best performance among the above three algorithms.

\section{Experiments}

To verify the theoretical development, experimental tests are performed in this section. The performances of the three self-sensing algorithms are compared. As for step I, a $\pm 4 \mathrm{~V} 10 \mathrm{kHz}$ sine wave is used as the input signal and the scaling factor is estimated by the three different algorithms. As for step II, the mechanical response in the time domain and the corresponding frequency spectrum are estimated. To show the effect of the scaling factor estimate error on the accuracy of the extracted mechanical response, different levels of the scaling factor estimate error are examined. Figure 1 (b) shows the actual experimental set-up except for the data acquisition system. To verify the results of the scaling factor estimate, the capacitance values of the PZT wafer and the feedback capacitor were measured by a commercial LCR meter, which had $0.05 \%$ accuracy. The dimension of the aluminum beam and the PZT wafer are $510 \mathrm{~mm} \times 19 \mathrm{~mm} \times 3 \mathrm{~mm}$ and $72.5 \mathrm{~mm} \times 19 \mathrm{~mm} \times 0.508 \mathrm{~mm}$, respectively. Table 1 shows the errors between the scaling factor measured from the LCR meter and the ones estimated from three different algorithms. As expected from the theoretical analysis, the orthogonality algorithm produces the smallest error.

\begin{tabular}{ccc}
\hline Estimation Algorithm & $S F$ & Relative Error $(\%)^{\mathrm{a}}$ \\
\hline RMS algorithm & -0.82745 & 0.122 \\
LMS algorithm & -0.82739 & 0.115 \\
Orthogonality algorithm & -0.82677 & 0.041 \\
\hline
\end{tabular}

${ }^{\text {a }}$ Note that $S F$ is the scaling factor estimated from the self-sensing circuit. And the $S F$ value estimated from the commercial LCR meter is used as the reference and its value is -0.82644 .

Table 1. Comparison of the three different self-sensing algorithms

In step II, a $\pm 4 \mathrm{~V}$ low-frequency chirp signal was used as the input signal. Figure 2 (a) and (b) show the input chirp signal in the time and frequency domains. In figure 2 (c) and (d), the output signal in the time and frequency domains from the self-sensing circuit is shown. Next, the scaling factor estimated from the LCR meter is perturbed 
to examine the effect of the scaling factor on the extracted mechanical response. The resonance frequencies of the test structure were clearly identified from the extracted mechanical response as shown in figure 2 (f). The error in the scaling factor estimate shifted the anti-resonance frequencies of the system horizontally. On the other hand, the resonance frequencies of the system were estimated properly.

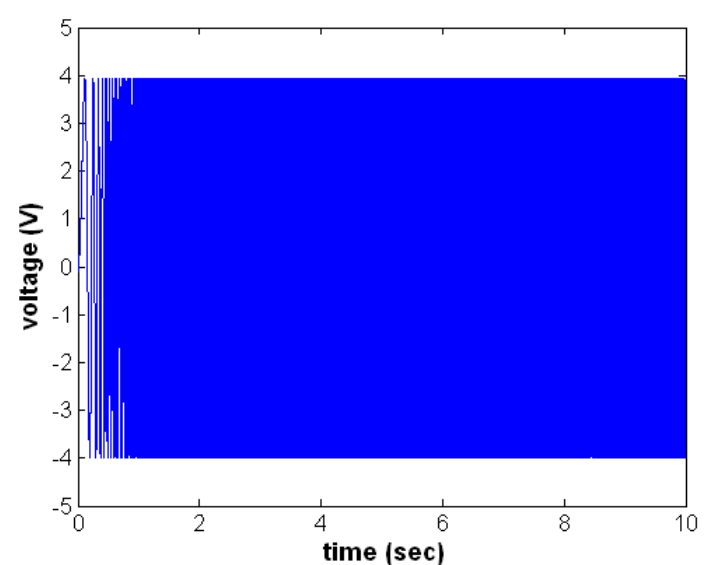

(a) A chirp input signal $(0-400 \mathrm{~Hz})$ in the time domain

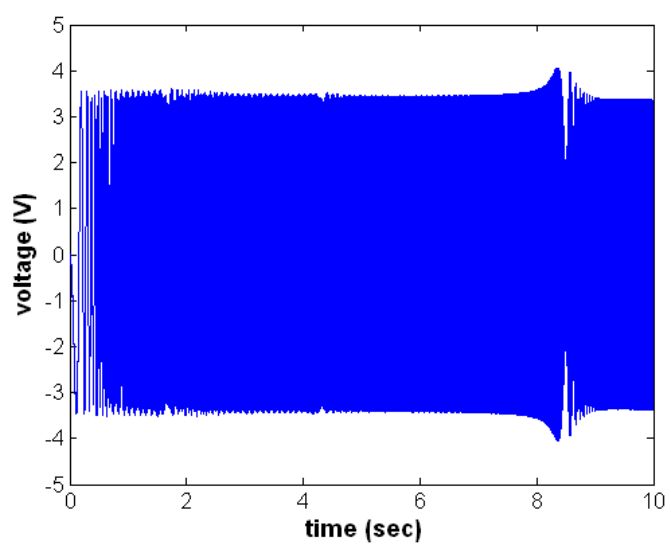

(c) The measured output voltage in the time domain

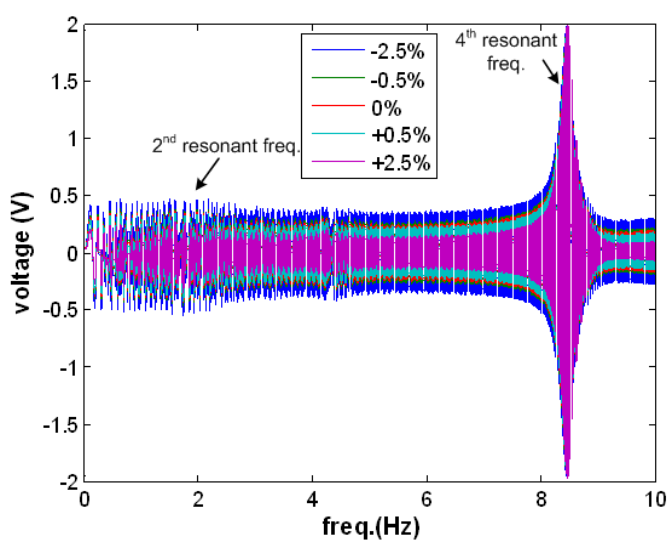

(e) The extracted mechanical time response

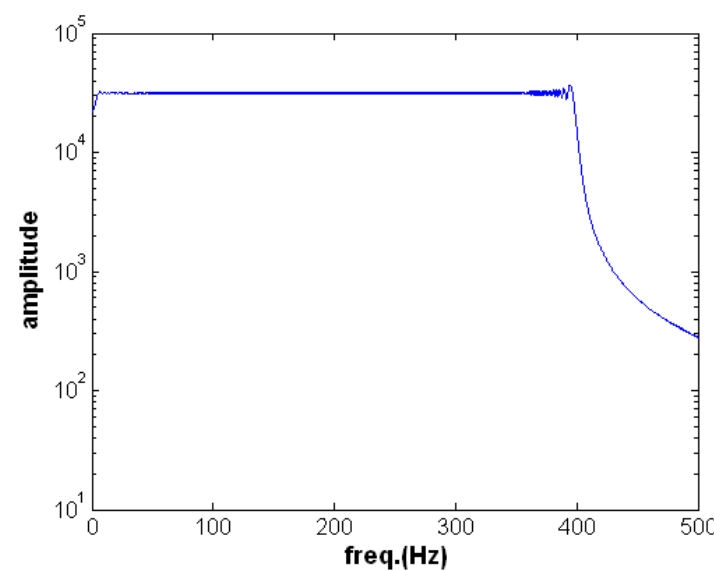

(b) The frequency content of the chirp signal

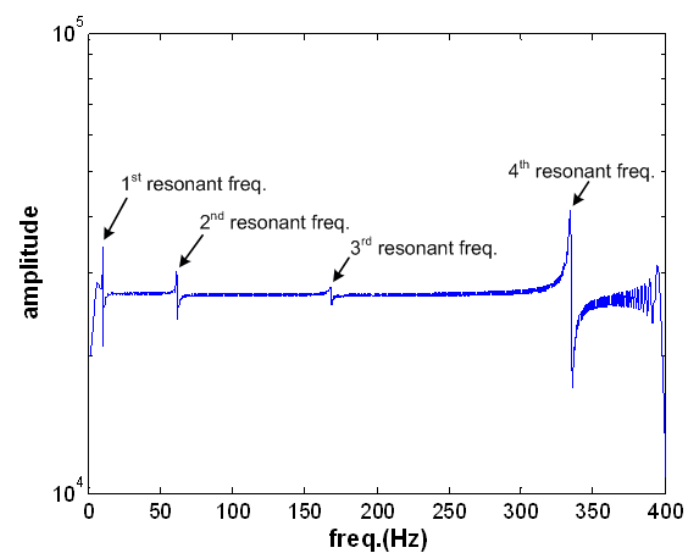

(d) The frequency spectrum of the output voltage

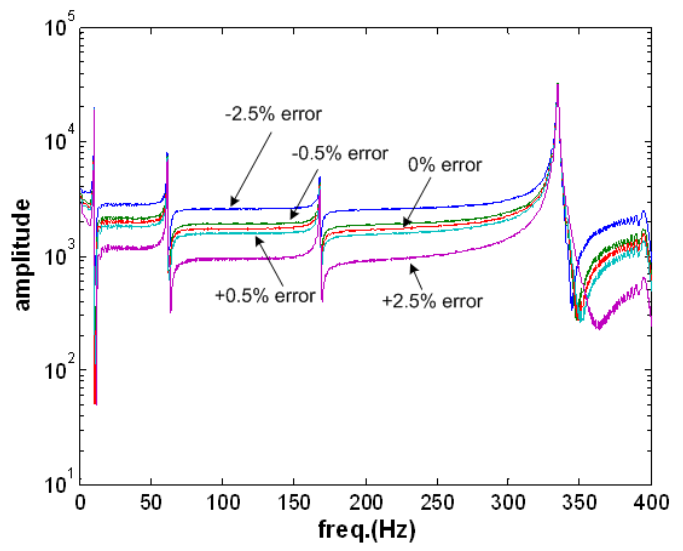

(f) The frequency spectrum of the extracted mechanical response

Figure 2. Estimated responses in the time and frequency domains when a chirp signal $(0 \sim 400 \mathrm{~Hz})$ is applied and the error level of the scaling factor is varied $\pm 2.5 \%$. 


\section{Conclusions}

In this study, a combination of self-sensing algorithms and a self-sensing circuit is developed particularly for active sensing devices used for guided wave generation and measurement. The goal of the proposed self-sensing is to use a single active sensing device such as PZT for simultaneous excitation and sensing of guided waves. A circuit analysis is performed to describe the relation between the PZT input and output voltages and the corresponding mechanical response voltage. In the first step, a specific probing input signal is applied to the PZT wafer to calibrate the self-sensing algorithm and to estimate the scaling factor. Then, the mechanical time response corresponding to an arbitrary input signal is extracted in the second step using the previously calibrated self-sensing algorithm. The feasibility of the proposed selfsensing scheme is demonstrated through experimental tests. The mechanical response of the system was successfully extracted in the time and frequency domains. Contrast to the previous self-sensing studies, the proposed self-sensing scheme emphasizes on accurate extraction of the mechanical response in the time domain. An ongoing research is underway to take advantage of the proposed self-sensing for developing sensor self-diagnosis schemes and structural damage diagnosis.

\section{Acknowledgements}

This work is supported by the Pennsylvania Infrastructure Technology Alliance (PITA), a partnership of Carnegie Mellon University, Lehigh University and the Commonwealth of Pennsylvania Department of Community and Economic Development.

\section{References}

Cole, D.G. and Clark, R.L., 1994, “Adaptive Compensation of Piezoelectric Sensoriactuators,” Journal of Intelligent Material Systems \& Structures, Vol. 5, pp. 665-672.

Dosch, J.J., Inman, D.J. and Garcia, E., 1992, “A Self-Sensing Piezoelectric Actuator for Collocated Control,” Journal of Intelligent Material Systems \& Structures, Vol. 3, pp. 166-185.

Fink, M., 1999, “Time-reversed acoustics,” Scientific American, Vol. 281, pp. 91-97.

Lee, S.J. and Sohn, H., 2006, “Active Self-sensing Module for Sensor Diagnosis and Structural Health Monitoring," Third European Workshop on Structural Health Monitoring, Granada, July 5-7, 2006

Sohn, H., Park, G., Wait, J.R., Limback, N.P. and Farrar, C.R., 2004, "Wavelet-based active sensing for delamination detection in composite structures," Smart Materials and Structures, Vol. 13, pp. 153-60.

Vipperman, J.S. and Clark, R.L., 1996, "Implementation of an Adaptive Piezoelectric Sensoriactuator," AIAA Journal, Vol. 34, pp. 2102-2109. 